Ann. Biol. anim. Bioch. Biophys., I975, 15 (4), 705-7I4.

\title{
ACQUISITION PAR L'OVOCYTE DE LAPINE ET DE VEAU DU FACTEUR DE DÉCONDENSATION DU NOYAU DU SPERMATOZOÏDE FÉCONDANT (MPGF)
}

\author{
C. THIBAULT, Micheline GLRARD et Y. MENEZO $\left.{ }^{(}\right)$ \\ Station de Physiologie animale et Université de Paris VI, \\ Centre national de Recherches zootechniques, I. N.R.A., \\ 78350 Jouy en Josas
}

\author{
RÉSUMÉ
}

La reprise complète de la méiose a été obtenue dans des ovocytes entourés des cellules du cumulus en culture. Ces ovocytes proviennent soit de follicules préovulatoires de Lapine, soit de follicules de taille moyenne $(\mathrm{I}, 5$ à $4,9 \mathrm{~mm})$ de Veaux femelles de 3 à 4 mois.

Dans ces conditions le MPGF n'est jamais présent dans l'ovocyte.

Seules de toutes les hormones utilisées dans les cultures, la prolactine et la testostérone facilitent la transformation du noyau du spermatozö̈de en un pronucléus mâle dans le quart des ovocytes. Il semble que cette action soit plus pharmacologique que physiologique.

Par contre, la culture de follicules entiers de Lapine, jusqu'à la thèque interne, en présence de gonadotropines, permet in vitro l'acquisition du MPGF par l'ovocyte qui peut être fécondé normalement et poursuivre un développement embryonnaire conduisant à des jeunes normaux.

La culture de follicules immatures de Veaux permet une reprise de la méiose dans les $3 / 4$ des ovocytes, mais dans 35 p. Ioo de ces ovocytes, clle s'arrête à la première division réductionnelle, tandis que les cellules du cumulus ne présentent pas la réaction de cissociation qui caractérise l'action des gonadotropines in vivo. Cultivées hors du follicule, ces mêmes cellules répondent aux gonadotropines. Le follicule immature est donc, soit imperméable aux gonadotropines, soit capable d'inhiber leur action.

Chez les Mammifères sous l'action du pic de gonadotropines, la maturation finale de l'ovocyte avant l'ovulation s'effectue toujours dans un follicule qui atteint sa taille maximum à la fois par multiplication cellulaire et sécrétion active des cellules de la granulosa. L'aptitude à sécréter différents stéroïdes sous 1'action de LH in vitro évolue au cours du cycle (SEAMARK, Moor et McINTOSH, I974) et le follicule préovulatoire, an moment même oì se produit la maturation de l'ovocyte, montre presque toujours une montée temporaire de la sécrétion d'estradiol, des androgènes et des progestines avant de se transformer en corps jaune.

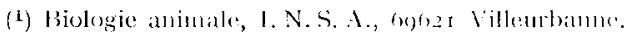


Chez les Amphibiens (Smith, Ecker et Subteiny, ig68; Schuerz, I972) et des Poissons (JAIABERT et Fostier, I974), les progestines sont nécessaires à la maturation nucléaire et cytoplasmique de l'ovocyte aussi bien in vivo qu'in vitro.

In vitro, chez les Mammifères, la reprise de la méiose ne nécessite l'intervention d'aucune hormone; elle est régulièrement obtenue simplement en soustrayant l'ovocyte et ses cellules attenantes du cumulus, à l'action répressive de la granulosa (Foote et Thibault, Ig69; Tsafriri et Channing, I975).

Nous avons même montré, chez la Irapine, que la chronologie de l'apparition des divisions méiotiques allant jusqu'à l'émission du premier globule polaire, était absolument parallèle in vivo, sous l'action des gonadotropines et in vitro sans aucun apport hormonal, la totalité des ovocytes atteignant la métaphase II en 9 heures enviton (Thibault, I972 ; Thibaul'T et GERard, I97 I, I973). Pour cet aspect de sa maturation nucléaire, l'ovocyte de Mammifère n'apparaît donc pas contrôlé de la même manière par les gonadotropines et les stéroïdes que l'ovocyte des Vertébrés inférieurs.

Mais, par ailleurs, nous avons découvert que les ovocytes de Lapine ayant accompli leur maturation nucléaire in vitro, bien que fécondables, sont inaptes à assurer la transformation très rapide du noyau condensé du spermatozoïde en un noyau somatique banal, le pronucleus mâle, ce qu'ont confirmé MoT'LIK et FuLKa (I974 b).

Rappelons que cette transformation se manifeste morphologiquement par un gonflement très rapide de la tête du spermatozoïde dès qu'ont fusionné les membranes des gamètes, histochimiquement par la transformation des protéines nucléaires du spermatozoïde, visualisée par la perte de l'arginine (KOPECNY et PAVLOK, I975), et fonctionnellement par la possibilité de replication de 1'ADN qui se produit quelques heures plus tard (OPREscu et Thibauis, I964).

Nous avons appelé MPGF le ou les facteurs présents dans le cytoplasne de l'ovocyte, qui assurent normalement ces transformations (ThIBAULT, 1972).

L'existence du MPGF paraît être un phénomène général, puisque l'absence d'évolution de la tête spermatique dans des ovocytes maturés in vitro a été retrouvée pour l'ovocyte de la Truie (Mo'TLIK et FULKA, I974 a) l'ovocyte humain (SOUPART, I975), et par nous-mêmes dans le présent travail, pour l'ovocyte de Vache.

Nous avons cherché, dans des systèmes totalement in vitro, à obtenir la maturation complète de l'ovocyte, incluant la présence du MPGF dans l'ovocyte cultivé, soit seulement entouré de ses cellules périovocytaires, soit à l'intérieur de follicules isolés de l'ovaire. Nous avons utilisé deux catégories de follicules et d'ovocytes : chez la I,apine les follicules préovulatoires, c'est-à-dire ceux qui peuvent répondre par une ovulation à l'action des gonadotropines, et chez le Veau, les nombreux follicules présents dans l'ovaire de l'animal impubère.

\section{MATÉRIEI, ET MÉTHODES}

Les techniques de préparation des ovocytes et des follicules ont été décrites précédemment pour la Lapine ('linibault et Geraris, I973).

Pour le Veau, l'animal est soumis avant abattáge, a un prétraitement combinant l'action d'un progestatif, l'acétate de fluorogestone penclant + jours et de 3 ooo l'I re PMSG administréc 20 à 40 heures avant l'abattage. Ceci permet d'obtenir par animal plusicurs dizaines de follicules de 1,5 à $4,5 \mathrm{~mm}$, ne présentant aucun signe d'atrésie, ni aucune activation folliculaire résultant 
le décharges endogènes de gonadotropines (ARRAU, 1974; Thibault, 1974). Lors de la préparation, les follicules atrétiques se reconnaissent à une vascularisation faible leur donnant un aspect grisâtre et à la présence de lébris cellulaires visibles par transparence dans le liquide folliculaire. Ils sont ainsi aisément éliminés.

Les follicules sont nettoyés des cellules interstitielles et de tout ou partie de la thèque externe. Ils sont placés en culture organotypique sur grille et millipore et le milieu ( $\mathbf{~ m l}$ ) est changé toutes les 24 heures.

Après de nombreux cssais, il est apparu que la stinulation du métabolisme des follicules par les gonadotropines et par la mise en culture entraînait une acidose interne, conduisant à l'atrésie, qu'il n'a été possible de compenser qu'en appauvrissant le milieu de culture par réduction ou suppression du sérum homologue, en augmentant son pouvoir tampon, en utilisant un pH élevé, compris entre 7,8 et 8 ,o et en augmentant l'apport d'oxygène.

Nous utilisons les milieux suivants :

Milieu $A$ : TC I99 (Earle), 70 parties, enrichi en pyruvate de Na à I 5 g/l, 5 parties, en potassium (bicarbonate, $\mathrm{r} 4 \mathrm{~g} / \mathrm{l}$, ro parties et additionné de sérum de Veau et de sérum de Veau fœetal en quantités égales, ro parties.

Le pyruvate et le potassium ont été ajoutés pour tenir compte de la composition du liquide folliculaire préovulatoire de Vache. Le rapport $\mathrm{Na} / \mathrm{K}$ est plus faible que dans les milieux conventionnels où il est voisin de celui du sérum.

Placé sous un mélange gazeux contenant soit 20 , soit $57 \mathrm{p}$. Ioo d'oxygène, 75 ou 38 p. Ioo d'azote et 5 p. Ioo de gaz carbonique, le $\mathrm{pH}$ de ce milieu est voisin de 7,8 . L'osmolarité est de $285^{-}$ 290 milliosmoles.

Milien $B$ : (tabl. I) La composition en aminoacides est identique à celle du liquide folliculaire (Menezo et Testart, r975). A l'exception de la glutamine et de la glycine, la concentration des différents aminoacides est plus faible que celle du milieu $\mathrm{A}$.

Les protéines du milieu A sont remplacées par de l'albumine sérique, à la concentration de Io $\mathrm{g} / \mathrm{l}$, qui ne contient aucun contaminant protéique ou minéral.

Le milieu ne renferme, à l'exception de la vitamine $\mathrm{C}$, aucune autre vitamine. Il ne contient ni nucléotides, ni bases puriques et pyrimidiques.

Les concentrations en glucose, pyruvate, lactate et le rapport $\mathrm{Na} / \mathrm{K}$ sont équivalents à ceux du milieu A. L'osmolarité ct les pouvoirs tampons sont voisins.

TABLEAU I

Milien de culture $B$

(en $\mathrm{mg} / \mathrm{I}$ oo $\mathrm{ml}$ )

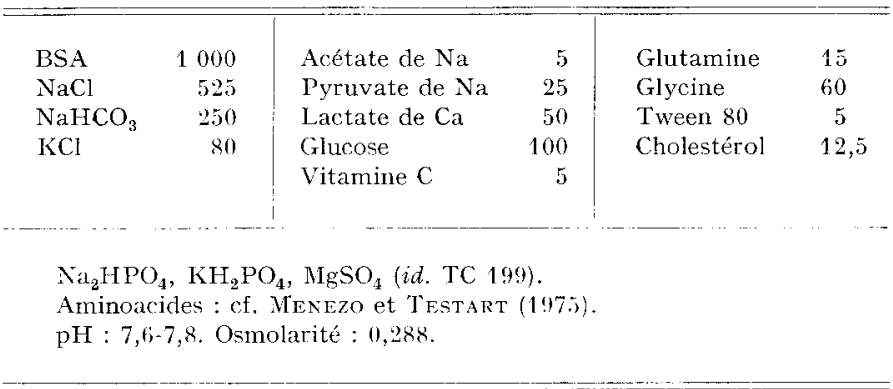

\section{RÉSUL'TA'TS E'T DISCUSSION}

\section{I. - Maturation in vitro de l'ovocyte de Veau isolé}

Cultivé sans hormone ou en présence de FSH, LH et estradiol-I $7 \beta$, pendant 23 à 30 heures, dans notre milieu $\mathrm{A}$, les ovocytes de Veau provenant de follicules de $\mathbf{I}, 5$ à $4,5 \mathrm{~mm}$ de diamètre reprennent pratiquement tous leur méiose, le plus souvent 
jusqu'à la métaphase II, que la teneur en oxygène du mélange gazeux soit de 5 ou de 20 p. IOO (tab1. 2). I,e pourcentage très élevé d'ovocytes atteignant la métaphase II, 94 p. Ioo dans ces expériences chez le Veau, roo p. roo avec l'ovocyte de Lapine adulte cultivé sans gonadotropines (ThrBaur,t, I972), quelle que soit la tension d'oxygène, en présence ou non de gonadotropines, laisse planer un doute sur l'intérêt des études telles que celles de JAGIELLO et al. (I975) sur le tôle d'hormones ou de médiateuts sur la maturation nucléaire de l'ovocyte in vitro.

TABLEAU 2

Maturation in vitro de l'ovocyte de Veau (Milieu A)

\begin{tabular}{|c|c|c|c|c|}
\hline \multirow{2}{*}{$\begin{array}{c}\text { Teneur en } \mathrm{O}_{2} \\
(\%)\end{array}$} & \multirow{2}{*}{ Hormones } & \multirow{2}{*}{$\begin{array}{c}\text { Nbre } \\
\text { ovocytes }\end{array}$} & \multicolumn{2}{|c|}{ Métaphase } \\
\hline & & & 1 & 2 \\
\hline 20 & $\mathrm{O}$ & 3 & 0 & : \\
\hline 20$)$ & ISH ou LH & 27 & 3 & 21 \\
\hline 20 & FSH, LH, li $17-\beta$ & $3 x$ & 2 & 36 \\
\hline ; & ISH, LH, F $17-\beta$ & 11 & 0 & 11 \\
\hline & & 79 & 5 & 71 \\
\hline
\end{tabular}

Sous l'action des gonadotropines, les cellules périovocytaires se dissocient, donnant 1'aspect caractéristique de corona radiata telle qu'elle apparaît in vivo quelques heures après la décharge ovulante.

'Transplantés dans une Vache en estrus, accouplés dans les heures précédant le transfert, ces ovocytes se sont révélés fécondables, mais ne possèdent pas de MPGF comme le montrent le tableau 3 et la figure I, puisque le noyau spermatique ne gonfle pas.

TABLEAU 3

Veau. Fécondation in vivo d'ovocytes maturés in vitro hors du follicule

\begin{tabular}{c|c|c|c}
\hline $\begin{array}{c}\text { Nombre d'oufs } \\
\text { récupérés }\end{array}$ & Non fécondés & $\begin{array}{c}\text { Pronucleus mâle } \\
\text { normal }\end{array}$ & $\begin{array}{c}\text { Fécondés } \\
\text { pas de MPGF }\end{array}$ \\
\hline 16 & 9 & $1\left({ }^{1}\right)$ & 6 \\
\hline
\end{tabular}

(1) Correspond à l'ovulation de la Vache receveuse.

Ces résultats confirment donc totalement ceux que nous avons obtenus chez la Lapine et les observations de Motrik et Fulka (I974 a) chez la Truie.

L'impossibilité d'obtenir la présence du MPGF dans les ovocytes accomplissant une maturation nucléaire normale in vitro nous a conduits à rechercher si ce facteur 
est synthétisé par l'ovocyte sous l'influence d'hormones ou de médiateurs sécrétés par le follicule sous l'action des gonadotropines ou si, produit par le follicule, il passe dans l'ovocyte par l'intermédiaire du liquide folliculaire.

\section{II. - Tentative d'induction du MPGF dans l'ovocyte de Lapine cultivé hors du follicule}

Après la décharge ovulante se produit une élévation rapide des stéroïdes caractérisée par une montée du niveau de l'estradiol, de la 20x-dihydroprogestérone et de la testostérone (HILLIARD et EATON, I97I; Hilíiard et al., I974). En outre, les hormones gonadotropes sont présentes à l'intérieur du follicule. Enfin, la décharge ovulante est accompagnée d'une décharge d'autres hormones, dont la prolactine.

Nous avons donc été conduits à rechercher in vitro si ces différentes hormones pouvaient induire l'élaboration du MPGF par l'ovocyte lui-même ou par les cellules périovocytaires (tabl. 4).

\section{TABIEAU 4}

Lssai dinduction du MPGF dans les wocytes de Lapine in vitro. Etat du noyau du spermatozoide 4-5) heures après fécondation

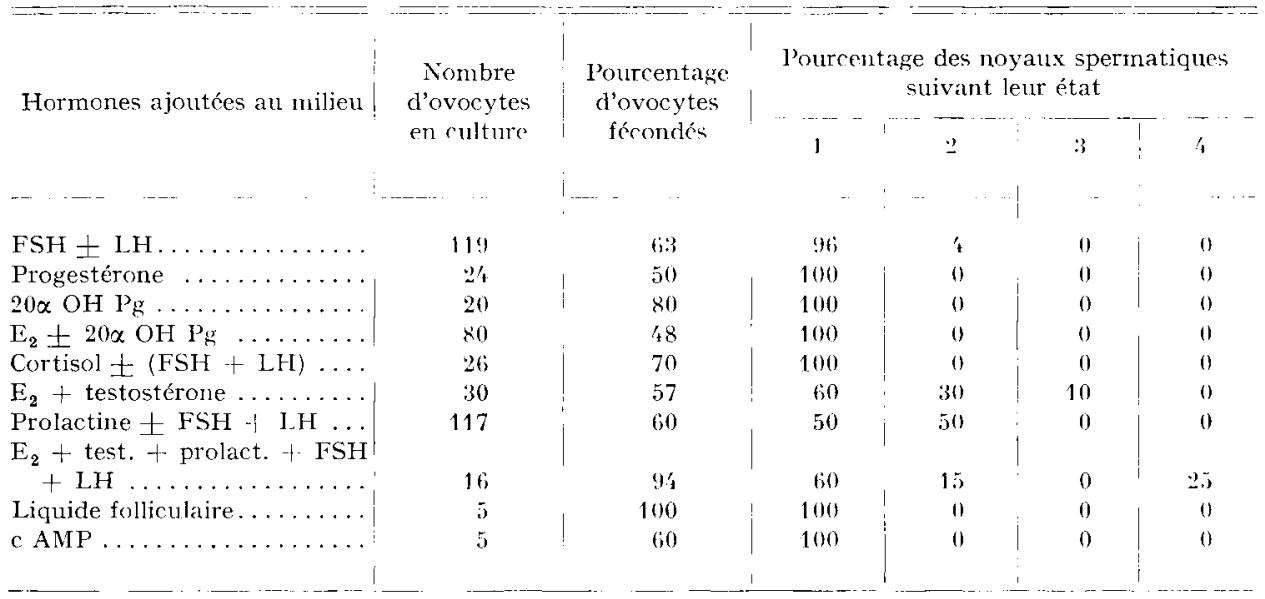

1. Pas d'évolution du noyau spermatique ou seulement formation d'une membrane.

2. Début de décondensation du noyau spermatique.

3. Formation d'un pronucleus mâle, mais des fragments du noyau spermatique restent présents.

'́. Pronucleus mâle normal.

Les hormones gonadotropes bien que provoquant la dissociation des cellules du cumulus, sont sans action.

L'estradiol et/ou la 20 $\alpha$-dihydroprogestérone, la progestérone, le cortisol, l'AMP cyclique, sont également inefficaces.

Par contre, la présence de prolactine et de testostérone facilite la formation du pronucleus mâle. Toutefois, cette formation est rarement normale, car le pronucleus formé contient plus ou moins de fragments non décondensés du noyau spermatique. 
Dans les rares cas où la décondensation a été complète, 4 à 5 heures après la fécondation (FSH, LH, P, T et $\hat{E}_{2}$ ), l'aspect réticulé du pronucleus mâle, après fixation, ne correspond pas exactement à l'aspect habituel du pronucleus dans les oufs normaux.

L'attention doit être attirée sur le fait que la présence de la prolactine, associée à la testostérone, entraîne environ $3^{\circ}$ p. Ioo de parthénogenèse spontanée durant la reprise de la méiose, avec segmentation régulière, ce qui laisse penser que ces hormones agissent plus en modifiant la perméabilité de l'ovocyte qu'en stimulant une synthèse physiologique du MPGF. Cependant, en utilisant une séquence hormonale d'estradiol, puis de $I 7 \alpha-O H$-progestérone, SouPART a obtenu le développement d'un pronucleus mâle dans les ovocytes humains maturés in vitro dans ces conditions (I974, I975).

\section{III. - Maturation intrafolliculaire in vitro de l'ovocyte de Lapine adulte}

Le maintien en parfait état, en culture, du follicule préovulatoire de Lapine pendant au moins 24 heures, condition absolue pour rechercher une maturation normale de l'ovocyte sous l'effet des gonadotropines, nécessite une oxygénation convenable. Celle-ci a été obtente, soit en utilisant l'air sous une pression de 5 à Io $\mathrm{kg}$, soit en utilisant un mélangegazeux contenant 55 p. Ioo d'oxygène. Le tableau 5 montre la double nécessité des gonadotropines et d'une oxygénation convenable.

TABLEAU 5

État des follicules de Lapine après $24 h$ de culture

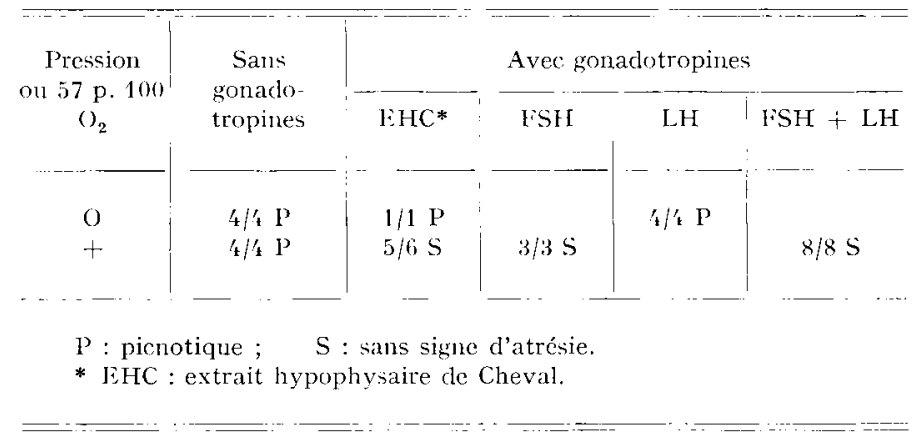

Dans ces conditions les ovocytes reprennent leur méiose et atteignent la métaphase II avec un retard de I à 2 heures environ par rapport à la maturation in vivo ce qui peut représenter le temps nécessaire à la pénétration des gonadotropines qui doivent atteindre le follicule par diffusion sans être véhiculées par le sang à l'intérieur de la thèque. Les cellules du cumulus se dissocient comme in vivo (fig. 3).

Les œufs maturés in vitro dans leur follicule possèdent le MPGF : l'action combinée de FSH et de LH permet d'obtenir une évolution du noyau spermatique absolument semblable à celle observée dans les ovocytes témoins normalement ovulés (Thibaul'r et GERARD, I973).

Deux Lapines ayant reçu de tels ovocytes, provenant de Lapines d'une autre 
race, ont donné naissance à des I,apereaux mâles et femelles qui, conduits à l'état adulte, se sont reproduits ('Thibault, Gerard et Menezo, I975).

La maturation dans le follicule préovulatoire est donc complète et normale in vitro en présence de gonadotropines.

\section{IV. - Maturation intrafolliculaire in vitro de l'ovocyte de Veau}

Chez les Mammifères à taux d'ovulation réduit, le nombre de follicules préovulatoires est faible et le but de techniques in vitro est précisément de pouvoir tenter d'utiliser des follicules de taille plus petite pour l'obtention d'un nombre élevé d'ovocytes normaux et fécondables. L'animal impubère présente l'avantage de posséder une population folliculaire beaucoup plus importante que l'adulte. C'est pourquoi nous avons utilisé le Veau de 3-4 mois. Le tableau 6 résume nos résultats.

\section{TABLEAU 6}

Cultures de follicules de Veau de 1,5 à $4,9 \mathrm{~mm}$

(Durée de culture 48 heures)

\begin{tabular}{|c|c|c|c|c|c|c|c|c|c|c|}
\hline \multirow{2}{*}{$\begin{array}{l}\text { Milieux } \\
\text { de culture }\end{array}$} & \multirow{2}{*}{$\begin{array}{l}\text { Nbre de } \\
\text { follicules }\end{array}$} & \multicolumn{2}{|c|}{ Granulosa } & \multicolumn{2}{|c|}{ Cumulus } & \multicolumn{3}{|c|}{ Etat nucléaire } & \multirow{2}{*}{ MI I $\underset{(\%)}{(\%)}$ M II } & \multirow{2}{*}{$\begin{array}{c}\text { M II } \\
(\%)\end{array}$} \\
\hline & & picn. & mit. & picn. & mit. & 1). $-\mathrm{R} M \mathrm{I}$ & $\mathrm{M} \mathrm{I}$ & MII & & \\
\hline A ou $B$ & 10 & $0-3 / 2$ & +10 & $0-3 / 2$ & $+\cdot 10$ & i) & 3 & 1 & $(40$ & 10 \\
\hline$A$ ou $B+I$ & 25 & $0-2 / 1$ & + & 0 & + & 21 & 3 & 0 & 14 & 0 \\
\hline$A E \pm I$ & 10 & $0-2-3$ & +10 & $0-1$ & +10 & 6 & 4 & 0 & 40 & 0 \\
\hline $\mathrm{A}$ oll $\mathrm{I} 3+\mathrm{GE}$ & 37 & $0-8$ & +10 & 0.1 & +10 & 14 & 11 & 12 & 62 & 32 \\
\hline$A$ oll $B+G I$ & 15 & 0 & + & 0 & + & 1 & 9 & 5 & 95 & 33 \\
\hline$A$ ou $B+G E I$ & 47 & $0-1$ & + & 0 & + & 7 & 19 & 21 & 8.5 & 65 \\
\hline
\end{tabular}

Milieu A ou B, voir Matériel et méthodes.

$G: o F S H$ et $\mathrm{oLH}: 1 \mu \mathrm{g} / \mathrm{ml}$ chacun ; E : Estradiol- $17 \beta: 100 \mathrm{ng} / \mathrm{ml}$

I : Insuline : $1 \mu \mathrm{g} / \mathrm{ml}$; Gaz : 57 p. $100 \mathrm{O}_{2}+38$ p. $100 \mathrm{~N}_{2}+5$ p. $100 \mathrm{CO}_{2}$.

État de picnose : 0 : pas de cellules picnotiques; 1 : quelques cellules picnotiques disséminées dans le tissu ; 2 : beaucoup de cellules picnotiques parmi les cellules saines ; $3:$ toutes les cellules sont picnotiques, dans au moins une région du follicule

Sans apport de gonadotropines, dans les milieux A ou B, la majorité des follicules présentent après 48 heures, des cellules picnotiques. Dans une échelle où o figure l'absence complète de cellules picnotiques, la valeur la plus fréquente est 2 . Cependant, quelques follicules demeurent complètement sains. Cette dégénérescence s'accompagne parfois d'une reprise de la méïose.

L'estradiol ajouté au milieu n'a aucun effet bénéfique sur l'état des cellules folliculaires : on observe également des cellules de la granulosa en voie de dégénérescence, ce qui se traduit également par une reprise spontanée de la méïose.

L'addition d'insuline améliore la survie des follicules et surtout permet le maintien d'une activité mitotique importante. 
Comme pour le follicule de I,apine, l'addition de gonadotropines dans les milieux, avec ou sans insuline, avec ou sans estradiol, permet la préservation complète de toutes les assises cellulaires et une reprise physiologique de la méiose : sur 99 follicules cultivés 48 heures, 77 p. Ioo ont repris leur méiose, dont plus de la moitié atteignent le stade final normal de la métaphase II.

Le tableau 6 paraît mettre en évidence un effet favorable de l'insuline sur 1'activité mitotique, sur le pourcentage d'ovocytes reprenant leur méïose (de 85 à 95 p. Ioo contre 62 p. Ioo sans insuline) et probablement sur le pourcentage d'ovocytes atteignant la métaphase II. Il n'apparaît aucun effet favorable de l'estradiol. Des conclusions définitives ne pourront être tirées que sur un plus grand nombre de cultures.

Le problème important que soulève cette étude est la proportion relativement faible des ovocytes qui atteignent la métaphase II après 48 heures (de 32 à 45 p. IOO).

Pour les follicules étudiés, dont la taille a varié de $\mathrm{I}, 2$ à $4,9 \mathrm{~mm}$ (70 p. Ioo se situant entre 2 et $3 \mathrm{~mm}$ ) nous n'avons pas trouvé de relation entre leur taille et la reprise plus ou moins complète de la méiose. Or, les ovocytes de follicules semblables reprennent pratiquement tous leur méiose (tab1. 2) quand ils sont cultivés hors de leur follicule. Cette absence de réponse suggère soit une imperméabilité du follicule immature aux gonadotropines, soit le maintien d'un contrôle par la granulosa de l'activité des cellules du cumulus dans le follicule immature. Efffectivement, on constate que la réaction caractéristique de dissociation des cellules du cumulus sous l'influence des gonadotropines, qui a lieu si on les cultive hors du follicule, ne se produit pratiquement pas à l'intérieur de celui-ci, (fig. 4). Si la non-dissociation des cellules du cumulus peut rendre compte de l'absence de reprise de la méïose, en l'état actuel de nos connaissances elle ne peut expliquer l'arrêt des divisions réductionnelles en métaphase I. L'arrêt en métaphase I a été rapporté pour des ovocytes de Souris en culture (Donahue, I968) et HaIDri, Mil, ER et GWatkin (I97I) et Gwatkin et HAIDRI (I974) ont montré, pour la Souris et le Hamster, que la proportion d'ovocytes atteignant in vitro la métaphase II, diminue quand la tension d'oxygène s'élève au-dessus de 5-ro p. Ioo. Il est possible que la proportion de $57 \mathrm{p}$. roo d'oxygène du mélange gazeux qui s'est avéré nécessaire pour assurer la survie des callules de la granulosa et du cumulus, soit trop élevée pour la maturation nucléaire complète de l'ovocyte.

Colloque D. C.R. S. T., Biologie de la Procréation,

Paris, $7-8$ mars 1975.

\section{REMERCIEMENTS}

Ce travail a bénéficié de l'aide de la D. G. R. S. T. (contrat no 74-7-05I4). Nous remercions M. Chesné, M. du Mesnil du Buisson, Mlle Catherine Siour et M. Testart pour l'aide apportée dans le travail réalisé avec les Veaux. 


\title{
SUMMARY
}

\author{
IN VITRO ACQUIRED ABILITY OF RABBIT \\ AND COW OOCYTE TO ENSURE SPERM NUCLEUS \\ DECONDENSATION DURING FERTILIZA'TION (MPGF)
}

Complete nuclear maturation of oocytes taken from rabbit and calf follicules may be generally obtained in vitro, even when the oocytes are from medium-size follicles. However, neither calf nor rabbit oocytes matured this way have MPGF, the factor transforming the sperm nucleus into the male pronucleus.

The culture of cumulus-clothed oocytes in presence of different hormones or mediators give the following results.

Progesterone, 2o $\alpha$-dihydroprogesterone, I $7 \beta$-estradiol, cortisol and the two gonadotropins are all without effect; after culture in presence of prolactin, as well as testosterone, partial dissociation of the sperm nucleus occurs. When prolactin, testosterone, estradiol and gonadotropins are combined, $25 \mathrm{p}$. Ioo of the oocytes form a subnormal male pronucleus.

When whole follicles of estrus rabbit are cultured in presnece of FSH and LH, absolutely normal oocytes are obtained which have MPGF. These oocytes, transplanted in to a host rabbit, give normal young.

Calf follicles survive and grow when cultured in modified TC I99, with Io p. Ioo of calf serum added, or in an entirely synthetic medium, with gonadotropins ; 77 p. roo of the oocytes resume meiosis. However, for some unknown reason, only 32 to +5 p. 1 oo of them reach m 2 ta jhas? II, while 95 p. Ioo of similar oocytes cultured outside of the follicles activate nuclear maturatio 1. This incomplete oocyte maturation is accompanied, in the follicle, by noi-disiociation of th, cumulus cells which normally occus in presence of FSH in vitro.

The permeability of immature follicles to gonadotropins and the interaction of diff $r$ : it cell types among themselves need for further studied.

\section{RÉFÉRENCES BIBLIOGRAPHIQUES}

Arrau J., 1974. Cinétique folliculaire et maturation ovocytaire chez le Veau traité par l'acétate de fluorogestone et la PMSG. Ann. Biol. anim. Bioch. Biophys., 14, 633-650.

Donahue R. P., r968. Maturation of the monse oocyte in vit"o. I. Sequence and timing of nuclear progression. J. exp. Zool., 169, $237-244$.

Foote W. D., Thibault C., I969. Recherches expérimentales sur la maturation in vitro des ovocytes de Truie et de Veau. Ann. Biol. anim. Bioch. Biophys, 9, 329-349.

GWATKIN R. B. L., FAidRi A. A., r974. Oxygen requirements for the maturation of Hamster oocytes. J. Reprod. Fert., $3 \%$, I27-I30.

Haidri A. A., Miller I. M., Gwatkin K. B. L., r97I. Culture of mouse oocytes in vitro, using a system without oil or protein. J. Reprod. Fert., 26, 409-4II.

Hilliard J., Eaton L. W, I97i. Estradiol-iz $\beta$, progesterone and $20 x$-hydroxypregn. 4 en - 3 one in rabbit ovarian plasma. II. From mating through implantation. Endocrinology, 89, 522-527.

Hilliard J., Pang C. N., Scaramuzzi R, J., Penardi R., Sawyer C. I1., Ig74. Secretion rates of estradiol, testosterone and prozesterone from right and left rabbit ovaries cannulated concurrently and successively. Biol. Reprod., 10, 364-369.

Jagiello G, Ducayen M., Miller W., Graffeo J., Fang I'. S., I975. Stimulation and inhibition with LH and other hormones of female mammalian meiosis in vitro. J. Reprod. Fert., 43, 9-22.

Jalabert B., Fostier A., I974. Action of gonadotropin and steroid hormones on maturation of Trout oocytes in vitro. Workshop on Oocyte maturation, Bruxelles, Société belge de Biologie cellulaire.

Kopecny V., PAvloK A., I975. Autoradingraphic study of mouse spermatozoan arginine-rich nuclear protein in fertilization. J. exp. Zool., 191, 85-95.

Menezo Y., Testard J., I975. Étude comparée du sérum sanguin et du liquide folliculaire préovulatoire chez la Vache. Ann. Biol. anim. Bioch. Biophys., 15, $\mathrm{I}-8$.

Notlik J., Fulka J., I974a. Fertilization of pig follicular oocytes cultivated in vitro. J. Reprod. Fert., 36, 235-237.

Motuk J., livika J, 1974 b. Fertilization and developnent in vivo of rabbit oocytes cultivated in vitro. J. Reprod. Fert., 40, 183-186.

Annale de Biologie animale. -- 1975 . 
Oprescu S., Thibault C., t965. Duplication de l'ADN dans les oufs de Lapine après la fécondation. Ann. Biol. anim. Bioch. Biophys., 5, I5I-156.

Schuetz A. W., I972. Hormones and follicular functions, 479-5 I I, in : Oogenesis, Bigcers J. and Schuetz A. W. ed. (University park Press, Baltimore).

Seamark R. F., Moor R. M., Mcintosh J. E. A., r974. Steroid hormone production by sheep ovarian follicles cultured in vitro. J. Reprod. Fert., 41, I43-I 58.

Smith L. D., Ecker R. E., SUbtelny S., r968. In vitro induction of physiological maturation in rana pipiens oocytes removed from their ovarian follicles. Inevelop. Biol., 17, 627.

Soupart P., I974. I'écondation humaine expérimentale : état de la question et perspective d'avenir. Bruxelles médical, 54, 473-499.

Soupart P., r975. In vitro maturation and fertilization of the human oocyte. In : La Fécondation, Thibault C. ed., Masson, Paris, 8r-93.

Tirbault C., r972. Final stages of mammalian oocyte maturation, 397-4II, in : "Oogenesis ", BIGGERS J. D. and Schuetz A W. Ed. (University Park Press, Baltimore).

Тнiвault C., I974. Conditions de maturation normale de l'ovocyte de quelques Mammifères in vivo et in vitro. Bruxelles médical, 54,619-628.

Thibault C., Gerard M., 197I. Maturation et fécondation in vitro de l'ovocyte de la Lapine, I2-25, in : Malformations congénitales des Mammifères, Tucimann-Duplessis H. Fd. (Masson, Paris).

Timbauta C., Gerard M., I973. Cytoplasmic and nuclear maturation of rabbit oocytes in vitro. Ann. Biol. anim. Bioch. Biophys., 13, Hors série, I45-155.

Thibault C., Gerard M., Menezo Y., 1975. Preovulatory and ovulatory mechanisms in oocyte maturation. J. Reprod. Fert., 45, (sous presse).

Tsafriri A., Channixg C., i975. An inhibitory influence of granulosa cells and follicular fluid upon porcine oocyte meiosis in vitro. Endocrinology., 96, 922-927.

\section{PI,ANCHE I}

FIG. I

Ovocyte de Veau, maturé in vitro, 5 heures après la fécondation in vivo. Absence de transformation du noyau du spermatozoïde.

FIG. 2

Fécondation normale d'un œuf de Vache, 5 heures environ après la pénétration du spermatozoide, son noyau s'est transformé en un pronucleus mâle (le pronucleus femelle est partiellement visible contre le pronucleus mâle).

FIG. 3

Follicule de Lapine en estrus cultivé I2 heures in vitro en présence de FSH et de $\mathrm{LH}_{\mathrm{H}}$. La réaction de dissociation des cellules du cumulus est nette et le premier globule polaire a été émis.

FIG. 4

Follicule de Veau cultivé $4^{8}$ heures en présence de gonadotropines. Le $2^{e}$ fuseau de maturation est visible, mais il n'y a pas de dissociation des cellules du cunulus qui ne se distinguent pas des cellules de la granulosa. Ians le coin droit en bas, partie de la thèque. 

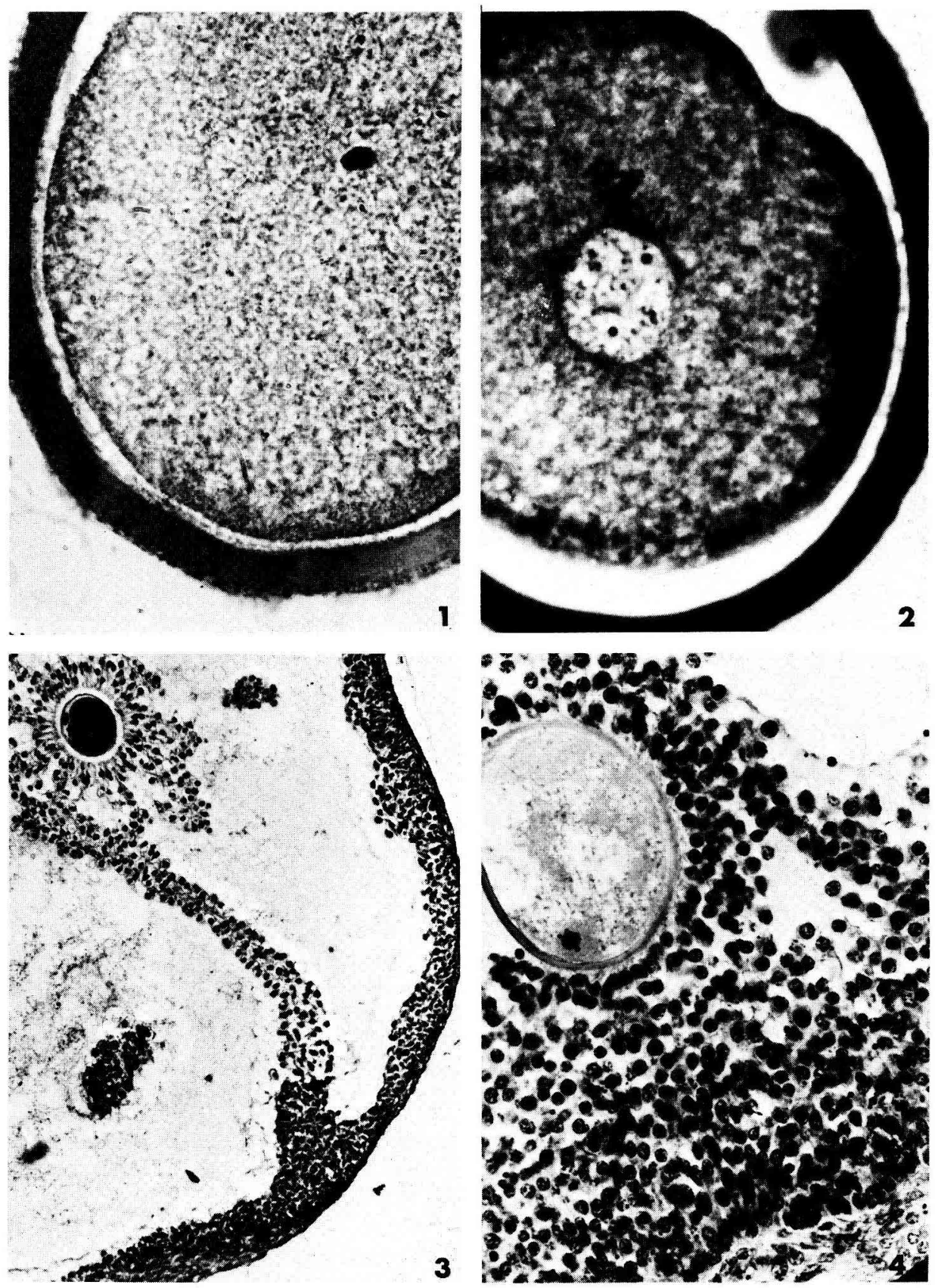

C. THIBAULT, Micheline GÉRARD et Y. MENEZO 